\title{
Vascular-Leukocyte Interactions
}

\section{Mechanisms of Human Decidual Spiral Artery Remodeling in Vitro}

\author{
Aleah D. Hazan, ${ }^{* \dagger}$ Samantha D. Smith, ${ }^{\ddagger}$ \\ Rebecca L. Jones, ${ }^{\ddagger}$ Wendy Whittle, ${ }^{\S}$ \\ Stephen J. Lye, ${ }^{\star \dagger}$ and Caroline E. Dunk ${ }^{\dagger}$ \\ From the Department of Physiology," University of Toronto, \\ Toronto, Ontario, Canada; the Research Centre for Women's and \\ Infants' Health Research Centre, ${ }^{\dagger}$ Samuel Lunenfeld Research \\ Institute, Mount Sinai Hospital, Toronto, Ontario, Canada; the \\ Maternal and Fetal Health Research Centre, ${ }^{\ddagger}$ University of \\ Manchester, Manchester Academic Health Science Centre, St. \\ Mary's Hospital, Manchester, United Kingdom; and the \\ Department of Obstetrics \& Gynaecology, ${ }^{\complement}$ University of Toronto, \\ Toronto, Ontario, Canada
}

Transformation of uterine spiral arteries is critical for healthy human pregnancy. We recently proposed a role for maternal leukocytes in decidual spiral artery remodeling and suggested that matrix metalloprotease (MMP) activity contributed to the destruction of the arterial wall. In the current study we used our first trimester placental-decidual co-culture (PDC) model to define the temporal relationship and test the mechanistic aspects of this process. PDC experiments were assessed by image analysis over a six-day time-course for degree of vascular transformation and leukocyte distribution around progressively remodeled arterioles. We observed rapid transformation in PDCs associated with loss of vascular smooth muscle cells, widening of the vessel lumen, and significant accumulation of uterine Natural Killer cells and macrophages within the vascular wall $(P<0.001)$ before trophoblast presence in the vessel lumens. These events did not occur in decidua-only cultures. Active MMP-9 was detected in leukocytes and vascular cells of remodeling arterioles, and inhibition of MMP-2/9 activity in PDC resulted in failure of decidual vascular remodeling compared with vehicle-treated PDCs. Apoptosis of vascular cells, macrophage-mediated phagocytosis, and vascular smooth muscle cell dedifferentiation contributed to the remodeling observed. The PDC model indicates that placental pres- ence is required to initiate decidual spiral artery remodeling but that uterine Natural Killer cells and macrophages mediate the early stages of this process at the cellular level. (Am J Pathol 2010, 177:1017-1030; DOI: 10.2353/ajpath.2010.091105)

After human blastocyst implantation, extravillous trophoblasts (EVTs) arise from placental villi and invade the decidualizing maternal endometrium (decidua) where they participate in the remodeling of spiral arteries. During remodeling, the spiral arteries undergo extensive changes including loss of their vasoactive medial vascular smooth muscle cells (VSMCs) and most of their intimal endothelial monolayer. This transforms the muscular, tightly coiled decidual spiral arteries into dilated sinusoids capable of increasing uterine blood volume to perfuse the placenta. This process is essential for successful establishment of utero-placental circulation and a healthy pregnancy. These changes are thought to be induced by the EVTs, which invade the spiral arteries, eventually reline the vessels, and acquire an endothelial-like phenotype. ${ }^{1}$ Failure of appropriate remodeling in the myometrial portions of these vessels has been described in patients with preeclampsia and intrauterine growth restriction. ${ }^{2}$

Before embryo implantation, the high progesterone levels of the late secretory phase initiate the first stages of

Supported by Canadian Institutes of Health Research \#MGC-13299, IHD\# 86232, and 165436, Tommy's the Baby Charity, and the NIHR Biomedical Research Centre. A.D.H. is supported by an Ontario Graduate Scholarship Master's Award and a CIHR Canada Graduate Scholarship Master's Award. S.D.S. is supported by a Medical Research Council/Tommy's the Baby Charity Studentship. Infrastructure support in Manchester was provided by Tommy's and the NIHR Biomedical Research Centre.

A.D.H. and S.D.S. contributed equally to this study.

Accepted for publication April 6, 2010.

Address reprint requests to Caroline E. Dunk, Ph.D., Research Centre for Women's and Infants' Health, Samuel Lunenfeld Research Institute, Mount Sinai Hospital, 25 Orde Street, Room 6-1025, Toronto, Ontario, Canada, M5T 3H7. E-mail: dunk@lunenfeld.ca. 
decidualization in the endometrium including angiogenesis of the spiral arteries and a large infiltration of innate immune cells. ${ }^{3}$ By early pregnancy, leukocytes comprise $40 \%$ of all decidual cells. Specialized uterine Natural Killer (UNK) cells and macrophages constitute $70 \%$ and $20 \%$ of decidual leukocytes, respectively. ${ }^{4,5}$ Both decidual macrophages and UNK cells produce angiogenic factors, including vascular endothelial growth factor, placental growth factor, and angiopoetin-2, which are proposed to contribute to decidual vascular remodeling. ${ }^{6,7}$ Similarly, a specific M2 tumor-associated macrophage population is thought to be the precipitating factor in tumor-mediated angiogenesis and metastasis as they possess many protumor activities including secretion of growth factors, matrix remodeling, and suppression of adaptive immunity. ${ }^{8,9}$ We suggest that the decidual macrophage may play a similar role in decidual angiogenesis and spiral artery remodeling. Multiple studies have identified an essential role for UNK cells in the murine implantation site. Mice deficient in either UNK or interferon- $\gamma$ signaling exhibit implantation abnormalities and defects of maternal artery remodeling. ${ }^{10-13}$ In humans, communication between uNK cell receptors and interstitial EVTs is believed to dictate depth of trophoblast invasion. ${ }^{14}$ However, no conclusive evidence exists to implicate UNK cells directly in human vascular transformation.

We recently reported an intimate relationship between uNK cells, macrophages, and remodeling arteries in biopsies of first trimester decidua basalis. ${ }^{15}$ Leukocytes were observed in close proximity to early and mid-stage remodeling arterial walls, in the absence of either interstitial EVTs (inEVTs) or endovascular EVTs (enEVTs). ${ }^{15}$ Moreover, we demonstrated that uNK cells and macrophages within the vascular wall expressed matrix metalloprotease (MMP)-7 and -9. MMPs are key proteases in the reproductive system and are known to be important for processes such as trophoblast invasion ${ }^{16-18}$ and focal degradation of the endometrial extracellular matrix during menstruation. ${ }^{19}$ We suggested that leukocyte-derived MMPs contribute to vascular remodeling, consistent with their reported roles in tumor angiogenesis ${ }^{20}$ and metastasis. ${ }^{21}$ However, in these in vivo specimens we were unable to conclusively describe the direct temporal relationship of the leukocytes with progression of vascular remodeling or directly test mechanistic functionality. We have developed a placenta-decidua coculture (PDC) model, adapted from previous work by Vicovac et al (1995), ${ }^{22}$ which allows us to study the dynamic processes of vascular remodeling in intact decidual explants. Using this model, we have previously reported EVT invasion into arterioles, endothelial cell and VSMC loss, and relining of arteries, but not veins, by endovascular EVTs. ${ }^{23}$

We hypothesized that uNK cells and macrophages participate in artery remodeling through specific mechanisms inducing vascular cell priming, destruction, and clearance. We used our PDC model to define the temporal, spatial, and mechanistic relationships of uNK cells and macrophages with spiral arteries during remodeling.

\section{Materials and Methods}

\section{Tissue Collection}

Placentae and decidua parietalis (without prior invasion) were obtained, following written informed consent from patients undergoing first trimester elective terminations at the Morgantaler Clinic and Mount Sinai Hospital (Toronto, Canada) or the Whitworth Clinic at St. Mary's Hospital (Manchester, UK). The Mount Sinai Hospital Research Ethics Board (Toronto) and the North West Research Ethics Committee (Manchester) approved collections of human tissues. Tissue was collected in cold PBS and dissected at 6 to 9 weeks $(n=16)$ according to the criteria of the Carnegie classification evaluating characteristics of embryonic/fetal parts.

\section{Human Placental-Decidual Co-Culture}

PDCs were established by placement of placental villi on the apical epithelial surface of patient-matched decidual explants. Briefly, small fragments of placental villi (15-20 mg wet weight) were dissected from the placenta, teased apart, and selected for the presence of EVT cell columns ensuring rapid attachment of the placental explant to the decidual surface. Thickness and integrity of decidua parietalis was assessed and dissected into 2 to $3 \mathrm{~mm}^{2}$ cubes. Decidual explants were placed with the apical epithelial surface uppermost in Millicell-CM culture dish inserts pore size $0.4 \mu \mathrm{m}$ (Fisher Scientific, Ottawa, Canada), pre-coated with $0.2 \mathrm{ml}$ undiluted phenol red-free Matrigel (Becton Dickinson, Mississauga, Canada). The Matrigel was allowed to solidify before the placement of the corresponding placental villous explant in contact with the decidual epithelial surface. Explants were cultured overnight with no media to allow attachment to occur followed by the addition of serum-free DMEMHam's F-12 media (Invitrogen, Burlington, Canada) supplemented with $20 \mathrm{ng} / \mathrm{ml}$ progesterone (Sigma, Oakville, Canada), $300 \mathrm{pg} / \mathrm{ml} 17 \beta$-estradiol (Sigma), and 100 $\mu \mathrm{g} / \mathrm{ml}$ normocin (Cedarlane laboratories, Burlington, Canada) at $3 \% \mathrm{O}_{2} / 5 \% \mathrm{CO}_{2}$. Culture media was changed every 48 hours. PDCs (from a single patient) were cultured in triplicate for each treatment point. Adjacent explants of decidua parietalis were cultured in the absence of placenta to confirm that there was no trophoblast invasion before the establishment of the culture and no degradation of blood vessels due to the culture conditions. Decidua-only controls from adjacent tissues and PDC were maintained in culture for 3 or 6 days.

\section{Fixation and Processing}

PDC replicates and matching decidua-only controls were fixed in $4 \%$ paraformaldehyde for 1 hour at room temperature and rinsed $3 \times$ in cold $\mathrm{Ca}^{2+}$ - and $\mathrm{Mg}^{2+}$-free PBS on a shaker for 20 minutes and stored in PBS at $4^{\circ} \mathrm{C}$ until processing. Explants were dehydrated by a gradient of ethanol in PBS solutions from $70 \%$ to $100 \%$. Experiments were cleared in xylene for 1 hour, excess xylene was removed by paraffin infiltration for 3 hours and embed- 
Table 1. Details of Antibodies Used for Immunohistochemistry

\begin{tabular}{|c|c|c|c|c|c|}
\hline Antibody & Species & Source & Dilution & Antigen retrieval & Specificity \\
\hline Cytokeratin & Mouse & Dako & $0.17 \mu \mathrm{g} / \mathrm{ml}$ & $\begin{array}{l}\text { Microwave: } 10 \mathrm{mmol} / \mathrm{L} \\
\text { Sodium }(\mathrm{Na}) \\
\text { Citrate } \mathrm{pH} 6\end{array}$ & $\begin{array}{l}\text { Epithelial cells } \\
\text { incl. } \\
\text { trophoblast }\end{array}$ \\
\hline CD31 (PECAM-1) & Mouse & Dako & $1.3 \mu \mathrm{g} / \mathrm{ml}$ & Microwave: NaCitrate & Endothelial cells \\
\hline$\alpha$-SMA & Mouse & Dako & $0.035 \mu \mathrm{g} / \mathrm{ml}$ & Microwave: NaCitrate & $\begin{array}{l}\text { Smooth muscle } \\
\text { cells }\end{array}$ \\
\hline CD45 & Mouse & Dako & $0.35 \mu \mathrm{g} / \mathrm{ml}$ & Microwave: NaCitrate & All leukocytes \\
\hline CD56 & Mouse & Dako & $0.3 \mu \mathrm{g} / \mathrm{ml}$ & Microwave: $1 \mathrm{mmol} / \mathrm{L}$ EDTA & uNK cells \\
\hline CD68 & Mouse & $\begin{array}{l}\text { Novocastra } \\
\text { Laboratories }\end{array}$ & $0.5 \mu \mathrm{g} / \mathrm{ml}$ & Microwave: NaCitrate & Macrophages \\
\hline MMP-9 & Mouse & Calbiochem & $4 \mu \mathrm{g} / \mathrm{ml}$ & Microwave: NaCitrate & MMP-9 \\
\hline $\begin{array}{l}\text { Lysozyme } \\
\text { muramidase }\end{array}$ & Rabbit & Biomedia & $1 \mu \mathrm{g} / \mathrm{ml}$ & Microwave: NaCitrate & Phagocytic cells \\
\hline $\begin{array}{l}\text { Anti-mouse } \\
\text { IgG-biotin }\end{array}$ & Goat & Dako & $0.024 \mu \mathrm{g} / \mathrm{ml}$ & Matched to primary antibody & Mouse IgG \\
\hline $\begin{array}{l}\text { Mouse IgG } \\
\text { Isotype }\end{array}$ & Mouse & Dako & Matched to primary & Matched to primary antibody & All IgG \\
\hline
\end{tabular}

ded in paraffin wax using an embedding machine. Only explants that had attached to the decidual epithelial surface by day 1 and remained attached at the time of collection and through fixation were processed. Occasionally the multiple solution changes during processing and paraffin-embedding resulted in the loss of the placental explant, as observed in some photomicrographs.

\section{Immunohistochemistry and Immunofluorescence}

Immunohistochemistry was performed on PDCs and decidua-only controls for vascular ( $\alpha$-smooth muscle actin [ $\alpha$-SMA], CD31), EVT (cytokeratin), leukocyte (CD45, CD56, CD68), and phagocytic (lysozyme muramidase) markers. Paraffin-embedded explants were sectioned to $5 \mu \mathrm{m}$ using a microtome, adhered to Superfrost ${ }^{++}$slides (Fisher Scientific) using a warm water bath and dried in an oven at $60^{\circ} \mathrm{C}$ overnight. Slides were deparaffinized in xylene and rehydrated through a gradient series of ethanol in PBS. Endogenous peroxidase activity was blocked by incubation of the sections in 3\% hydrogen peroxide (Fisher Scientific) in methanol for 40 minutes. Antigen retrieval methods specific for each antibody are summarized in Table 1. All slides were incubated with Dako protein blocking solution (Dako, Mississauga, Ontario, Canada) for 1 hour at room temperature to block nonspecific binding. Usage conditions, source, and specificity for all immunohistochemistry primary and secondary antibodies are provided in Table 1. Slides were developed using the labeled streptavidin biotin - horseradish peroxidase (1 hour) (Dako) and 3,3-diaminoben- zidine $^{+}$in diluting solution (Dako). After counterstaining with Harris Hematoxylin Solution (Sigma) slides were dehydrated in an ascending ethanol series, cleared in $x y$ lene, and mounted with Permount (Fisher Scientific).

To determine the identity of MMP- $9^{+}$cells, dual immunofluorescence was performed using combinations of antibodies as described in Table 2. Sections were dewaxed, rehydrated, and antigens retrieved as indicated in Table 1. Autofluorescence was blocked using sodium borohydride (Sigma, Gillingham, UK) applied at $0.1 \%$ in TBS for $3 \times 10$ minutes. Nonimmune block $(10 \%$ goat serum [Sigma, UK] and $2 \%$ human serum [in-house] in $0.1 \%$ Tween-20 [BioRad, Hemel Hempstead, UK] in Tris buffered saline) was applied before incubation with primary antibody for 1 hour at $37^{\circ} \mathrm{C}$. Antigen was detected by rabbit anti-mouse FITC conjugate (Dako, Ely, UK) at $46 \mu \mathrm{g} / \mathrm{ml}$. Unlabeled goat anti-mouse IgGs (Dako, UK) were applied at $15.2 \mu \mathrm{g} / \mathrm{ml}$ for 1 hour to saturate binding site for the first primary antibody. Nonimmune block was reapplied before incubation with the second primary antibody for 1 hour at $37^{\circ} \mathrm{C}$, which was then detected by application of rabbit anti-mouse Alexa Fluor568 conjugate (Molecular Probes, Paisley, UK) at 40 $\mu \mathrm{g} / \mathrm{ml}$. Sections were mounted using Vectashield containing DAPI (Vector Laboratories, Burlingame, CA). Negative controls included combinations of mouse and rabbit IgGs to match concentration of primary antibodies, omission of all antibody to control for autofluorescence, or omission of the first secondary antibody to control for cross reactivity between the first primary and the second secondary antibodies.

Table 2. Combinations of Antibodies Used for Dual Immunofluorescence

\begin{tabular}{clll}
\hline First primary & First secondary & Second primary & Second secondary \\
\hline TUNEL reagent & N/A & $\alpha$-SMA & Anti-mouse Alexa Fluor-568 \\
TUNEL reagent & N/A & CD31 & Anti-mouse Alexa Fluor-568 \\
MMP-9 & Anti-mouse-FITC & CD56 & Anti-mouse Alexa Fluor-568 \\
MMP-9 & Anti-mouse-FITC & $\alpha$-SMA & Anti-mouse Alexa Fluor-568 \\
MMP-9 & Anti-mouse-FITC & Pan cytokeratin & Anti-mouse Alexa Fluor-568 \\
MMP-9 & Anti-mouse-FITC & CD31 & Anti-mouse Alexa Fluor-568 \\
\hline
\end{tabular}

N/A, not applicable. 
To determine whether remodeling vessels contained apoptotic cells, terminal deoxynucleotidyl transferase biotin-dUTP nick end labeling (TUNEL) assays were performed using the In Situ Cell Death detection kit with FITC detection (Roche, Welwyn Garden City, UK) as described previously. ${ }^{15} \mathrm{~A}$ positive control was treated with DNase I for 10 minutes at $37^{\circ} \mathrm{C}$, while a negative control was generated by the omission of the terminal deoxynucleotidase enzyme. To determine the identity of apoptotic cells, sections were TUNEL stained, followed by immunofluorescent detection of anti- $\alpha$-SMA or anti-CD31 using rabbit anti-mouse Alexa Fluor-568 conjugate.

\section{In Situ Zymography}

In situ zymography to detect gelatinase activity in PDC was performed as previously described. ${ }^{15}$ Unfixed PDCs $(n=3)$ were embedded in OCT and snap frozen in liquid nitrogen. Ten micrometer sections were cut on a cryostat (Leica, Milton Keynes, UK), mounted on Superfrost ${ }^{++}$ slides, fixed with $10 \%$ normal buffered formalin for 30 minutes, and stained with $\alpha$-SMA and CD45 to identify remodeling arteries with leukocytic infiltration. In situ zymography was then performed on serial sections to examine MMP activity. Freshly cut air-dried $10 \mu \mathrm{m}$ sections were fixed in $10 \%$ normal buffered formalin for 5 minutes at $4^{\circ} \mathrm{C}$. Slides were washed and counterstained with propidium iodide for 8 minutes. One hundred microliters of the substrate, DQ gelatin ( $25 \mu \mathrm{g} / \mathrm{ml}$, Invitrogen, Paisley, UK) which fluoresces when cleaved, was layered over the tissue section, covered with a coverslip, and incubated for 16 hours at $37^{\circ} \mathrm{C}$. Negative and positive controls were included; 1,10 phenanthroline (Invitrogen, UK) or collagenase was applied to control sections for 1 hour at $37^{\circ} \mathrm{C}$ before counterstaining.

\section{MMP-2/9 Inhibition}

Given the MMP-9 expression in VSMCs, endothelial cells, UNK cells, and macrophages, as well as the active MMP-2/9 detected in VSMCs and leukocytes in both in vivo decidua basalis and PDC model specimens, we decided to use the PDC model to investigate MMP-2/9 function. This was performed by manipulating culture conditions with addition of a specific MMP-2/9 inhibitor, (2R)-[(4-Biphenylylsulfonyl)amino]-N-hydroxy3-phenylproprionamide (Calbiochem, Darmstadt, Germany), more commonly referred to as MMP-2/9 Inhibitor II, to determine the role of these proteases on vascular remodeling processes.

To minimize damage to the decidual epithelial surface decidual explants were immobilized in a Petri dish and injected at 2 basal stromal sites with $50 \mathrm{nmol} / \mathrm{L} \mathrm{MMP-2/9}$ Inhibitor II or with vehicle (0.1\% DMSO; Sigma) (50 $\mu \mathrm{l} /$ site) using a 700 series Microliter Hamilton syringe with cemented needle gauge 22s (VWR, Mississauga, Ontario, Canada) followed by a 30 minute incubation at $37^{\circ} \mathrm{C}$ before establishment of PDC as described above (MMP Inh: $n=3$, vehicle: $n=3$ ). The concentration used in this study more selectively inhibits MMP-9 over MMP-2.

\section{Digital Image Capture}

Photomicrographs of immunohistochemical staining were captured using an Olympus BX61 Upright Microscope and an Olympus DP70 12.5 megapixel camera with accompanying Olympus software (Olympus America Inc., Center Valley, PA). Images of immunofluorescent staining were captured using a Zeiss fluorescence microscope with an AxioCam MRn (Zeiss, Welwyn Garden City, UK).

\section{Quantification of Vascular Remodeling and Leukocyte Distribution by Image Analysis}

Serial sections of PDCs and corresponding deciduaonly controls from day 3 and 6 of culture immunostained for $\alpha$-SMA, CD45, CD56, and CD68 were scanned at $\times 16$ and $\times 100$ magnification (Visiopharm Integrator System Version 3.0.8.0, Visiopharm, Horsholm, Denmark). Adjacent digital photomicrographs were stitched together automatically by the Visiopharm software to create composite images of the entire PDC tissue section. Image analysis was conducted using Visiopharm's Visiomorph analysis software which established pixel classification parameters based on pigment for stroma, positive immunohistochemical stain, and vessel lumen. These parameters were then applied to each image and PDC decidua were divided into 3 depths (0-500, 500-1000, and 1000-2000 $\mu \mathrm{m})$ from the epithelial surface. Veins and glands were excluded from the analysis based on morphological assessment after immunostaining for the vascular markers CD31 and $\alpha$-SMA. To identify changes in the decidual arterioles, areas of VSMCs and vessel lumens were calculated by Visiomorph within each depth and expressed as a ratio ( $\alpha$-SMA/lumen). For each patient sample, triplicate $\alpha$-SMA/lumen measurements from replicate PDCs were calculated and averaged.

In addition, we determined leukocyte association with arterioles in decidua-only controls and in PDCs before and during remodeling. Decidual arterioles were designated by morphology as unremodeled (multiple, wellorganized layers of smooth muscle, intact endothelium, and narrow lumen), actively remodeling (disrupted VSMC and/or disorganized medial VSMC and endothelial desquamation and/or loss), or advanced remodeling (complete loss of VSMC, few/no endothelial cells, and dilated lumen) arterioles. On average the medial wall thickness of unremodeled arterioles from both PDCs and deciduaonly controls was $\sim 15 \mu \mathrm{m}$. A line was drawn to denote the lumen of each vessel and concentric rings were automatically executed at $15 \mu \mathrm{m}$ distances from this line by the Visiopharm software. The concentric zones 30 to $60 \mu \mathrm{m}$ from vessel lumens were examined during optimization, and no significant differences in area of CD $45^{+}$ leukocytes was found between the 15 to $30 \mu \mathrm{m}$ zone and those further from vessels. Measurements taken $>30 \mu \mathrm{m}$ from the vessel lumen frequently included leukocytes clustered around neighboring vessels. Therefore, only the two $15 \mu \mathrm{m}$ concentric distances closest to the arterioles were used in the comparisons presented. This 
ensured that the area examined accounted for the muscular arterial wall and excluded leukocytes not directly associated with the vessel under investigation. Immunohistochemical staining was classified by pixel pigment (as described above) to calculate area of leukocyte staining (CD45, CD56, and CD68), which was then expressed as a proportion of each concentric ring area (ie, area of $\mathrm{CD} 45^{+} / 0$ to $15 \mu \mathrm{m}$ concentric ring area) and excluded the arteriole lumen area.

\section{Statistics}

Because of the nonparametric nature of our data, statistical analyses were performed using Kruskal-Wallis Analysis of Variance with Dunn's post hoc test. Data are presented as medians and interquartile ranges in box and whisker plots. Whiskers of vascular remodeling quantification include $100 \%$ of the data. Whiskers of leukocyte quantification represent $10 \%$ to $90 \%$ of data in-
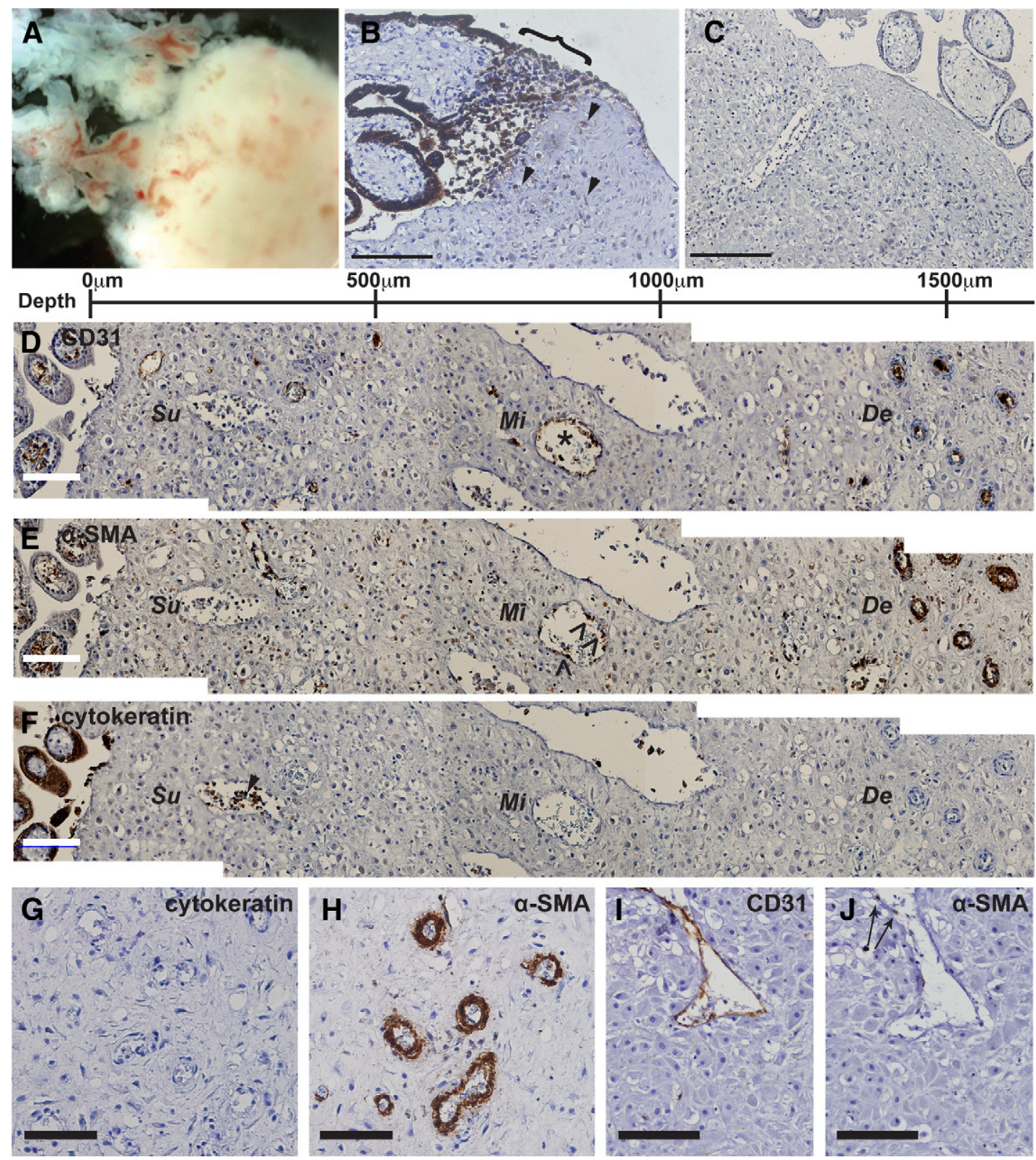

Figure 1. Decidual spiral artery remodeling in the placental-decidual co-culture model. A: Representative photomicrograph of a PDC. B: An EVT column (cytokeratin) attaching to the decidual epithelial surface (bracket). A few interstitial EVTs (arrowheads) are observed in the superficial decidual stroma arising from a well-attached EVT cell column. C: Isotype control. D-F: Representative serial sections from a day 6 PDC shown with scale of decidual depth. Progressive remodeling occurs along an artery in the direction of trophoblast (F: cytokeratin) invasion (left to right). The segment of the artery at the superficial epithelial decidual surface $(S u)$ contains EVTs in the lumen (arrowhead, F) and is more fully remodeled. Early vascular remodeling is observed in the mid-segment of the arteriole (Mi) by endothelial cell (D: CD31, asterisk) swelling and disruption, and VSMC loss (E: $\alpha$-SMA, open arrowheads) in the absence of trophoblast. Deep vessels ( $D e$ ) demonstrate no evidence of remodeling. $\mathbf{G}$ and $\mathbf{H}$ : Intact arterioles from the superficial portion of decidua-only cultures have no trophoblast present (G: cytokeratin) and possess multiple layers of vascular smooth muscle (H: $\alpha$-SMA). I and J: Veins were excluded from analysis by morphology. Serial sections demonstrate a thin endothelial layer (I: CD31) and sporadic VSMC (J: $\alpha$-SMA, arrows). Scale bars $=100 \mu \mathrm{m}$. 
clusive. Additional comparisons were made using MannWhitney $t$-tests as indicated.

\section{Results}

\section{Temporal Vascular Remodeling in Human PDC}

The time course of decidual vascular changes induced by presence of placental explants was examined (Figure 1A). At the site of placental-decidual contact, cytokera$\mathrm{tin}^{+}$trophoblast were consistently observed in anchoring columns arising from the tips of placental villi and attaching to the decidual epithelial surface (Figure 1B). Serial sections of a representative PDC at day 6 demonstrated progressive remodeling along a vessel from the point of placental contact (Figure 1, D: CD31; E: $\alpha-S M A$; and F: cytokeratin). Superficial portions of arterioles (within the first $500 \mu \mathrm{m}$ from the decidual epithelial surface) showed features of more advanced remodeling (Figure 1: right of Su) than deeper portions of the same vessels, evidenced by little or no remaining endothelium, disorganized, sporadic VSMCs, and intraluminal EVTs. Mid-portions of the arterioles showed evidence of active remodeling in the absence of intraluminal EVTs (Figure 1: Mi). Active remodeling is characterized by endothelial desquamation and shedding into the vessel lumen (Figure 1D: *), as well as disruption and loss of VSMCs (Figure 1E: ${ }^{\wedge}$ ). Deep arterioles (Figure 1: De) in PDCs remained largely untransformed, with intact VSMCs and endothelial layers, and no EVT invasion, similar to decidua-only control arterioles (Figure 1, G and $\mathrm{H}$ ). Veins were excluded based on morphological features such as thin layers of VSMCs, intact flattened endothelium, and dilated lumens (Figure $1, \mathrm{I}$ and $\mathrm{J}$ ). All arterioles in decidua-only control cultures were intact and unremodeled.

Quantification of vascular remodeling in PDCs at day 3 and 6 along with corresponding decidua-only controls is presented as median and interquartile ranges in Figure 2. Decreased $\alpha$-SMA/lumen ratios resulted from the loss of VSMCs and dilation of the remodeling arteriole lumens. No statistical differences in $\alpha$-SMA/lumen ratios for decidua-

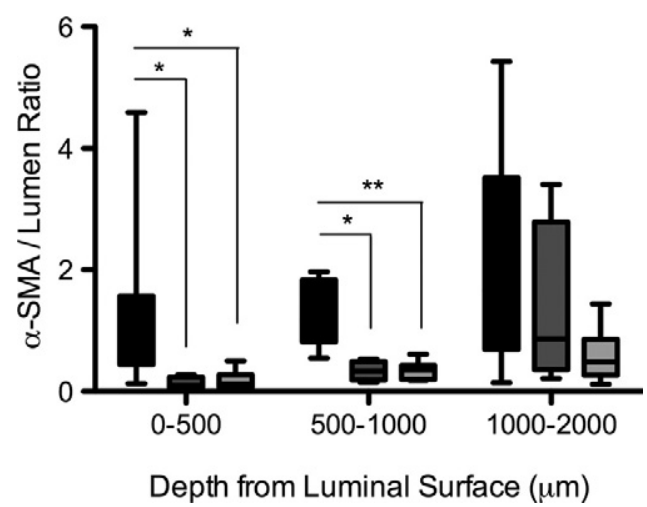

Figure 2. Quantification of vascular remodeling over a 6-day time-course. Image analysis quantification of $\alpha$-SMA/lumen area ratios showed a significant decrease at increasing depths from the PDC decidual epithelial surface at both day three (dark gray bars: $n=5$ ) and six (light gray bars: $n=6$ ) as compared with pooled day three and six decidua-only controls (black bars: $n=9) .{ }^{*} P<0.05,{ }^{*} P<0.01$. only controls were observed between 3 and 6 days, so the data were pooled for graphical representation. Within 500 $\mu \mathrm{m}$ of the decidual epithelial surface, a tenfold decrease in the $\alpha$-SMA/lumen ratio of PDCs at both day $3(P<0.05)$ and $6(P<0.05)$ was observed compared with decidua-only controls. No significant difference existed between day 3 and 6 PDCs at this first depth, indicating that maximal VSMC loss occurs within the first 3 days in culture. The $\alpha$-SMA/lumen ratio between 500 to $1000 \mu \mathrm{m}$ was also significantly decreased at both day $3(P<0.05)$ and $6(P<$ 0.01 ) compared with decidua-only controls, with no significant difference between time points. Beyond $1000 \mu \mathrm{m}$ there was a trend toward a decrease in $\alpha$-SMA/lumen ratio with time in culture, using pooled decidua-only controls. Interestingly, when $\alpha$-SMA/lumen ratios from day 6 PDCs were compared with their internal experiment-matched day 6 controls at the 1000 to $2000 \mu \mathrm{m}$ depth, a significant decrease in the PDC $\alpha$-SMA/lumen ratio was found $(P<0.05)$. Similar comparison of day 3 PDCs to their internal experimental controls demonstrated no difference, indicating a progressive, ongoing process of remodeling over time in these cultures.

\section{Leukocyte Association with Vessels Differed with Progression of Remodeling}

Decidual spiral arterioles 25 to $50 \mu \mathrm{m}$ in diameter from PDCs (day 3 and 6) were designated as unremodeled, early, active, or advanced remodeling based on the characteristic changes in vascular cells (Figure 3). Unremodeled arterioles possessed an intact CD31 ${ }^{+}$endothelial lining (Figure 3A), three to four layers of $\alpha-\mathrm{SMA}^{+} \mathrm{VSMC}$ (Figure 3B), and no evidence of cytokeratin ${ }^{+}$trophoblast (Figure 3C). Early and active remodeling arterioles were characterized by endothelial cell swelling, desquamation, and sloughing off into the lumen (Figure 3, E and I). These arterioles exhibited varying degrees of VSMC hypertrophy, disorganization, and loss (Figure 3, F and J) with no evidence of EVTs (Figure 3, G and K). These changes were not observed in veins, which possessed an intact flattened endothelium and a single smooth muscle layer (Figure 3, E and F). Advanced remodeling arterioles were observed within the first $500 \mu \mathrm{m}$ depth of the decidua at day 6 and were relined with EVTs (Figure 30). Minimal endothelium and VSMCs remained within the vascular wall of these arterioles (Figure 3, M and N). Throughout remodeling distinct changes in leukocyte distribution were observed. While leukocytes were abundant in the stroma, these cells were excluded from the walls of unremodeled arterioles (Figure 3D). In the early stages of remodeling, leukocytes cluster around the arterioles compared with their stromal distribution (Figure $3 \mathrm{H}$ ). Within the wall of actively remodeling arterioles, a large infiltration of $\mathrm{CD}_{4} 5^{+}$leukocytes was observed (Figure 3L). Fewer leukocytes were detected in proximity to advanced remodeling arterioles (Figure 3P).

\section{Uterine NK Cells and Macrophages Infiltrated Actively Remodeling Spiral Arterioles}

Leukocytes $\left(\mathrm{CD}^{2} 5^{+}\right)$subclassified as $\mathrm{CD}^{+} 6^{+}$uNK cells and $\mathrm{CD} 68^{+}$macrophages were observed infiltrating the 


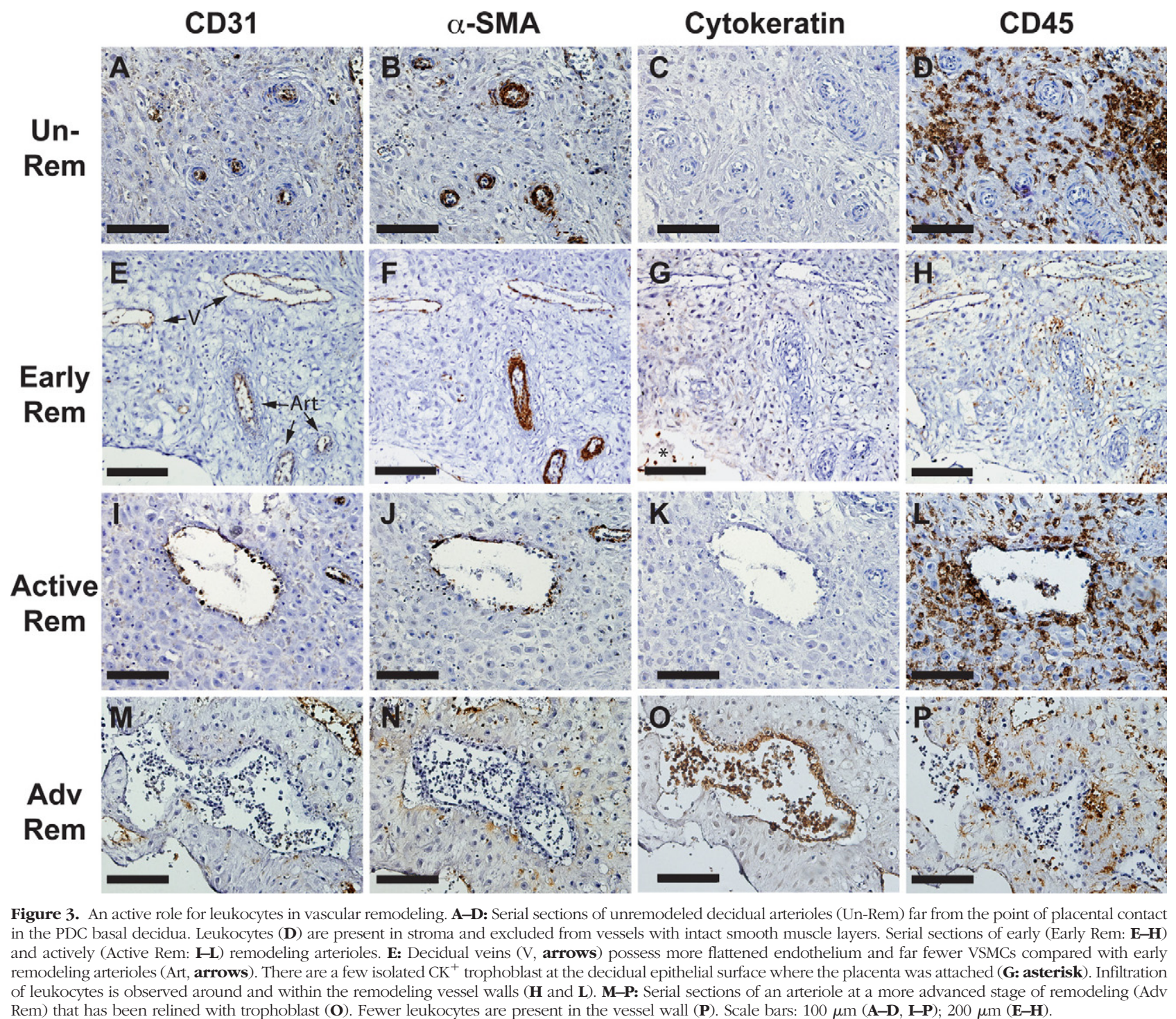

vascular walls of actively remodeling arterioles by immunohistochemistry (Figure 4). Remodeling arterioles with some remaining VSMC layers (Figure 4A) contained CD45 ${ }^{+}$leukocytes (Figure 4B) including uNK cells (Figure $4 C$ ) and macrophages (Figure 4D) around and within their vascular walls. Increased disruption of VSMC (Figure $4 \mathrm{E})$ was associated with denser infiltration of leukocytes (Figure 4F), uNK cells (Figure 4G), and macrophages (Figure $4 \mathrm{H}$ ), many of which crossed the endothelium and accumulated in the lumen of actively remodeling arterioles. Leukocytes did not infiltrate decidua-only control arterioles with intact VSMC (Figure 4, I-L).

\section{Quantification of Leukocyte Association with Decidual Arterioles}

By day 3, the superficial portions of the arterioles $(<1000$ $\mu \mathrm{m}$ from the decidual epithelial surface) exhibited characteristics of active remodeling. Similar observations were made in larger, deeper spiral arterioles/arteries (>1000 $\mu \mathrm{m}$ from the decidual epithelial surface) at day 6. Remodeling arterioles were compared with unremodeled arterioles deeper within the PDCs and in superficial portions of decidua-only controls. Positive stain for leukocytes (CD45) was expressed as a proportion of the total concentric zone area around them (Figure 5). On quantification, no significant differences in the area of $\mathrm{CD}_{4}{ }^{+}$staining surrounding decidua-only control arterioles compared with untransformed PDC arterioles were found (Figure 5, A and B). There were, however, significantly increased proportions of $\mathrm{CD}_{4} 5^{+}$staining within the 0 to $15 \mu \mathrm{m}$ zone of actively remodeling arteriole lumens at both day 3 (Figure 5A: $n=$ 31 arterioles) and 6 (Figure 5B: $n=27$ ) compared with arterioles from decidua-only cultures ( $d 3 n=20 ; d 6 n=25)$ or unremodeled arterioles from the same experiment (d3 $n=28$; d6 $n=22)(P<0.001$ for all). In more advanced remodeling arterioles containing trophoblast ( $d 6 n=8)$, the proportion of $\mathrm{CD}_{4} 5^{+}$staining in the 0 to $15 \mu \mathrm{m}$ zone was decreased as compared with actively remodeling vessels within the same depth and from the same experiments ( $n=$ 


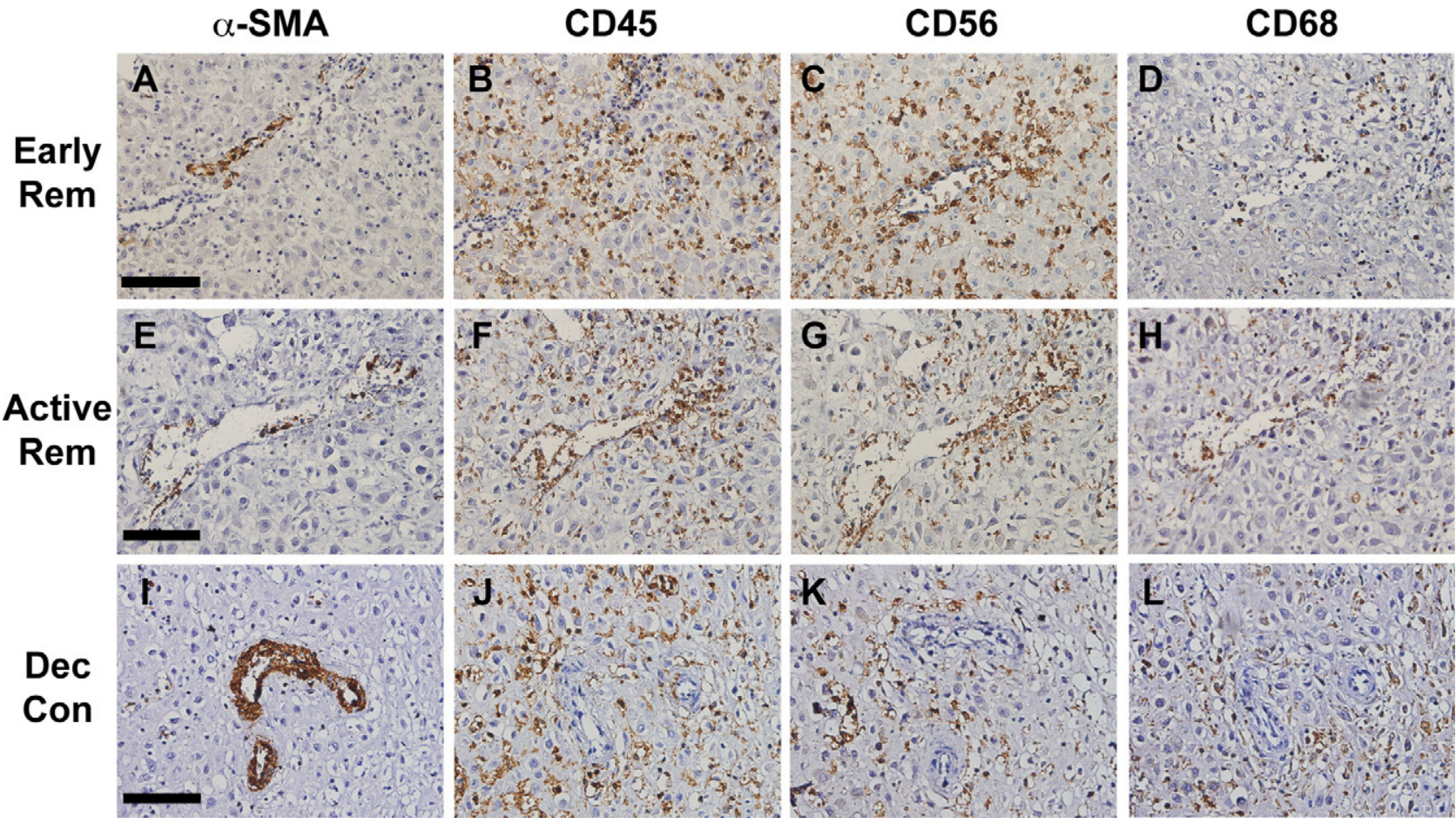

Figure 4. Leukocyte subtypes involved in active vascular remodeling. A-D: Serial sections of an example vessel in an early stage of remodeling (Early Rem). Vessel possesses a single remaining layer of smooth muscle (A). The majority of leukocytes (B) in close association with the vessel are CD $56^{+}$uNK cells $(\mathbf{C})$ with a few $\mathrm{CD}_{68}{ }^{+}$macrophages (D). E-H: Serial sections of an arteriole in a more progressed stage of remodeling (Active Rem). VSMCs are disrupted, sporadic, and shed into vessel lumen (E). Leukocytes are densely clustered within the arterial wall and lumen (F). Many uNK cells (G) and macrophages (H) are present in arteriole wall and lumen. I-L: Serial sections of a decidua-only control vessel (Dec Con). Intact vessel possesses multiple layers of smooth muscle (I). Both uNK cells $(\mathbf{K})$ and macrophages $(\mathbf{L})$ are present in the decidual stroma but do not demonstrate a close association with the vessel. Scale bars $=100 \mu \mathrm{m}$.

27, $P<0.05$ ) (data not shown). There were no significant differences in area of $\mathrm{CD}_{4} 5^{+}$staining between the 15 to 30 $\mu \mathrm{m}$ zones around decidua-only controls, unremodeled, early remodeling, or advanced remodeling arterioles at day 3 or 6 .

Image analysis was used to quantify proportion of positive CD56 (uNK cells) and CD68 (macrophages) staining surrounding vessels similar to that performed for the common leukocyte antigen marker (CD45). Substantial and significant increases in both uNK cells and macrophages within the 0 to $15 \mu \mathrm{m}$ zone of actively remodeling arterioles compared with either decidua-only control arterioles (Figure 5, C and D: CD56 d3\&6 $P<0.001$; E and F: CD68 d3 $P<0.01$, d6 $P<0.001)$ or unremodeled PDC arterioles (CD56 d3\&6 $P<0.001$; CD68 d3\&6 $P<0.001)$ were observed. At day 3 , no differences existed in $\mathrm{UNK}$ cell and macrophage proportions in the outer (15-30 $\mu \mathrm{m}$ ) zone between the different remodeling stages. By day 6 , more macrophages were observed in the outer zones of actively remodeling vessels compared with either decidua-only controls $(P<0.05)$ or unremodeled PDC arterioles $(P<0.01)$.

\section{MMP-9 Expression During Vascular Remodeling}

MMP-9 expression by uNK cells, macrophages, vascular cells, and EVTs was examined by dual immunofluorescence (Figure 6). VSMCs of early remodeling arterioles (Figure 6, A-C) were MMP-9 ${ }^{+}$, as were CD56 ${ }^{+}$uNK cells infiltrating actively remodeling arterioles (Figure 6, E and
F). Furthermore, the enEVTs present in advanced remodeling arterioles (Figure 6, G-I) were also MMP-9+ ${ }^{+}$. Decidual stromal, glandular epithelial, and endothelial cells were MMP-9 $^{+}$(data not shown). No staining for MMP-9 was detected in the negative control (Figure 6J). In situ zymography revealed MMP-2/9 (gelatinase) activity in infiltrating leukocytes associated with remodeling arterioles identified by CD45 stained serial sections (Figure 6K, enlarged in L). MMP-2/9 activity was also observed within the VSMCs of the disrupted vascular wall identified by $\alpha$-SMA-stained serial sections (Figure 6, $\mathrm{M}$ and $\mathrm{N}$, enlarged in $\mathrm{O}$ ).

\section{MMP-2/9 Inhibition Impairs Decidual Vascular Remodeling in Co-Culture}

To investigate the role of MMP-2 and -9 in vascular remodeling, decidual explants were microinjected with MMP-2/9 Inhibitor II (50 nmol/L) or vehicle (0.1\% DMSO) and cultured with placenta for four days. Remodeling of the superficial arterioles at the epithelial surface was observed in the vehicle-injected control PDCs (Figure 7B) exhibiting similar loss of $\alpha$-SMA to that seen in PDCs with no treatment. In PDCs injected with MMP-2/9 Inhibitor II, however, the arterioles remained unremodeled with multiple intact VSMC layers although the placental explant remained in contact (Figure $7 \mathrm{C}$ ). Decidual leukocytes $\left(C D 45^{+}\right)$did not infiltrate arterioles of the MMP-2/9 Inhibitor II-injected PDCs (Figure 7E) while they did in vehicle-injected controls (Figure 7D). Image analysis demonstrated 
A

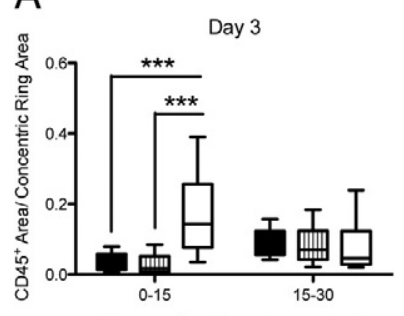

Distance from Vessel Lumen ( $(\mu \mathrm{m})$

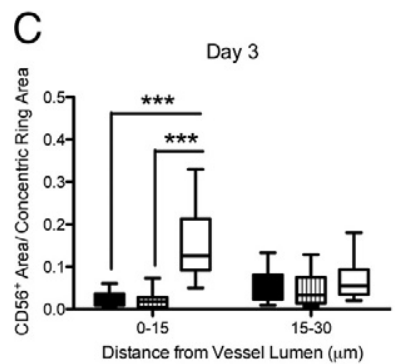

$\mathrm{E}$

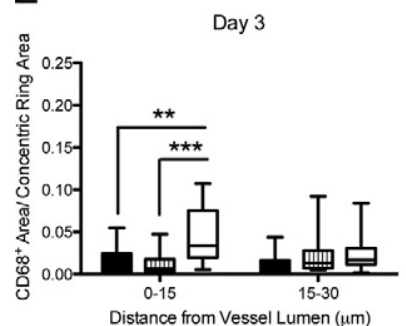

B

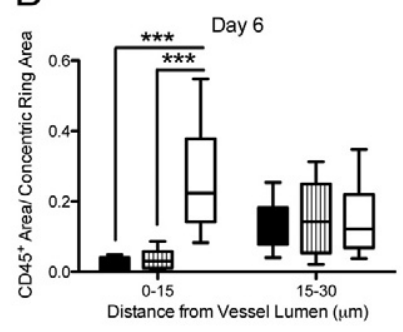

D Day 6

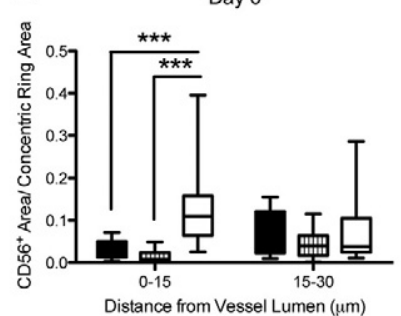

$\mathrm{F}$

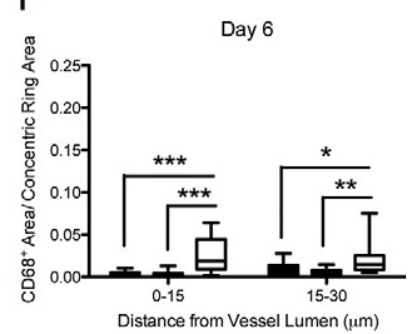

Figure 5. Quantification of leukocytes associated with remodeling arterioles and unremodeled arterioles from PDCs and decidua-only controls. Black bars: Decidua-only control culture arterioles (day 3: $n=20$, day 6: $n=25$ ) Striped bars: Unremodeled arterioles from PDCs (day 3: $n=28$, day 6: $n=$ 22). White bars: Actively remodeling arterioles from PDCs (day 3: $n=31$, day 6: $n=27$ ). Leukocyte infiltration was assessed by area of CD 45, CD56, or CD68 staining and expressed as a ratio of the concentric ring areas $0-15$ $\mu \mathrm{m}$ and $15-30 \mu \mathrm{m}$ from arteriole lumens. A significant increase in CD $45^{+}$ leukocyte/concentric ring area was observed at the $0-15 \mu \mathrm{m}$ distance on both days 3 (A) and 6 (B). A significant increase in the proportion of uNK cells in the $0-15 \mu \mathrm{m}$ ring surrounding actively remodeling vessels was observed at day 3 (C) and 6 (D). A significant increase in the proportion of macrophages within the $0-15 \mu \mathrm{m}$ ring surrounding actively remodeling vessels was observed at both day $3(\mathbf{E})$ and $6(\mathbf{F})$. In contrast to day 3 , a significant increase in the proportion of macrophages within the 15-30 $\mu \mathrm{m}$ zone around actively remodeling arterioles was observed at day $6(\mathbf{F})$. ${ }^{*} P<0.05,{ }^{* * *} P<0.01,{ }^{* * * * *} P<0.001$

significantly greater $\alpha$-SMA/lumen ratios within both 0 to 500 $\mu \mathrm{m}(P<0.01)$ and 500 to $1000 \mu \mathrm{m}(P<0.05)$ depths of MMP-2/9 Inhibitor II-injected PDCs compared with vehicleinjected PDC controls (Figure 7A; $n=3$ ).

\section{Evidence for Apoptosis and Phagocytosis of Vascular Cells During Remodeling}

Potential mechanisms contributing to the rapid, progressive loss of both endothelium and VSMCs were investigated. Apoptotic cells were identified by positive TUNEL stain in the wall of actively remodeling arterioles as both endothelial cells (CD31; Figure 8, A, B, and E) and VSMCs ( $\alpha$-SMA; Figure $8, \mathrm{C}, \mathrm{D}$, and E) by dual immunofluorescence. Toluidine blue staining of semithin sections showed apoptotic vascular cells displaying condensed pyknotic nuclei. Large granular leukocytes (likely uNK cells) were observed within the arteriole lumen and macrophages were embedded in the vascular wall (Figure 8F).

In early remodeling arterioles/arteries (>500 $\mu \mathrm{m}$ from the decidual epithelial surface), we observed VSMC dispersion and reversion of phenotype, characterized by a gradual decline in $\alpha$-SMA immunostaining of cells at increasing distances from the remaining intact rings of VSMCs (Figure 8G). Additionally, these arterioles displayed accumulation of macrophages and clusters of lysozyme muramidase ${ }^{+}$cells, indicating phagocytosis of peripheral vascular cells (Figure $8, \mathrm{H}$ and $\mathrm{I}$ ).

\section{Discussion}

In this article we present novel data of vascular-leukocyte interactions and potential mechanisms mediating early human decidual spiral artery remodeling. In the PDC remodeling was initiated in arterioles close to the site of placental contact (epithelial decidual surface) and progressed along the length of the vessels. By day 3 of culture, EVTs were present in the superficial portions of the arterioles, however vascular remodeling and significant leukocyte infiltration and accumulation was observed in deeper portions of the same arterioles. This was accompanied by decreased $\alpha$-SMA/lumen ratios, suggesting a reduction in the thickness of VSMC layers and/or widening of the lumen. In contrast, $\alpha$-SMA/lumen ratios were similar at all depths in decidua-only controls confirming that the decreased $\alpha$-SMA/lumen ratios in superficial PDC decidua were induced by the presence of the placental explant. In more advanced remodeling arterioles demonstrating significant loss of VSMC and endothelial cells and relined by enEVTs, there were significantly fewer leukocytes within the perivascular area suggesting a transient involvement with remodeling arterioles during the period of vascular cell loss. Thus, our PDC model demonstrates that early decidual vascular remodeling occurs before enEVT presence and is associated with a large infiltration of both uNK cells and macrophages.

This conclusion serves to elaborate on the earlier dogma that credits invasion of EVTs to be primarily responsible for "physiological transformation" of the spiral arteries. Much of these data come from placental bed biopsies, including the decidual-myometrial junction, and decidual basalis specimens where VSMC disruption is observed in the presence of both inEVTs and enEVTs. ${ }^{24,25}$ In our PDC model we routinely observed invasion of enEVTs into arterioles, not inEVTs into stroma. This is likely due to the inability of EVTs to penetrate the intact decidual epithelial surface. ${ }^{26}$ They may selectively differentiate to enEVTs because they can more easily access the arteriole lumens by either migrating down arterioles that open to the dissected edge of the explant or enter arteriole openings at the decidual epithelial surface, which are reported to occur as early as 30 to 40 days post coitum in decidua basalis. ${ }^{3,27}$ Both of these routes of invasion are observed in our PDC model. While this model does not account for the role of inEVTs in vascular remodeling, these results indicate that enEVTs are sufficient to initiate a process similar to in vivo vascu- 

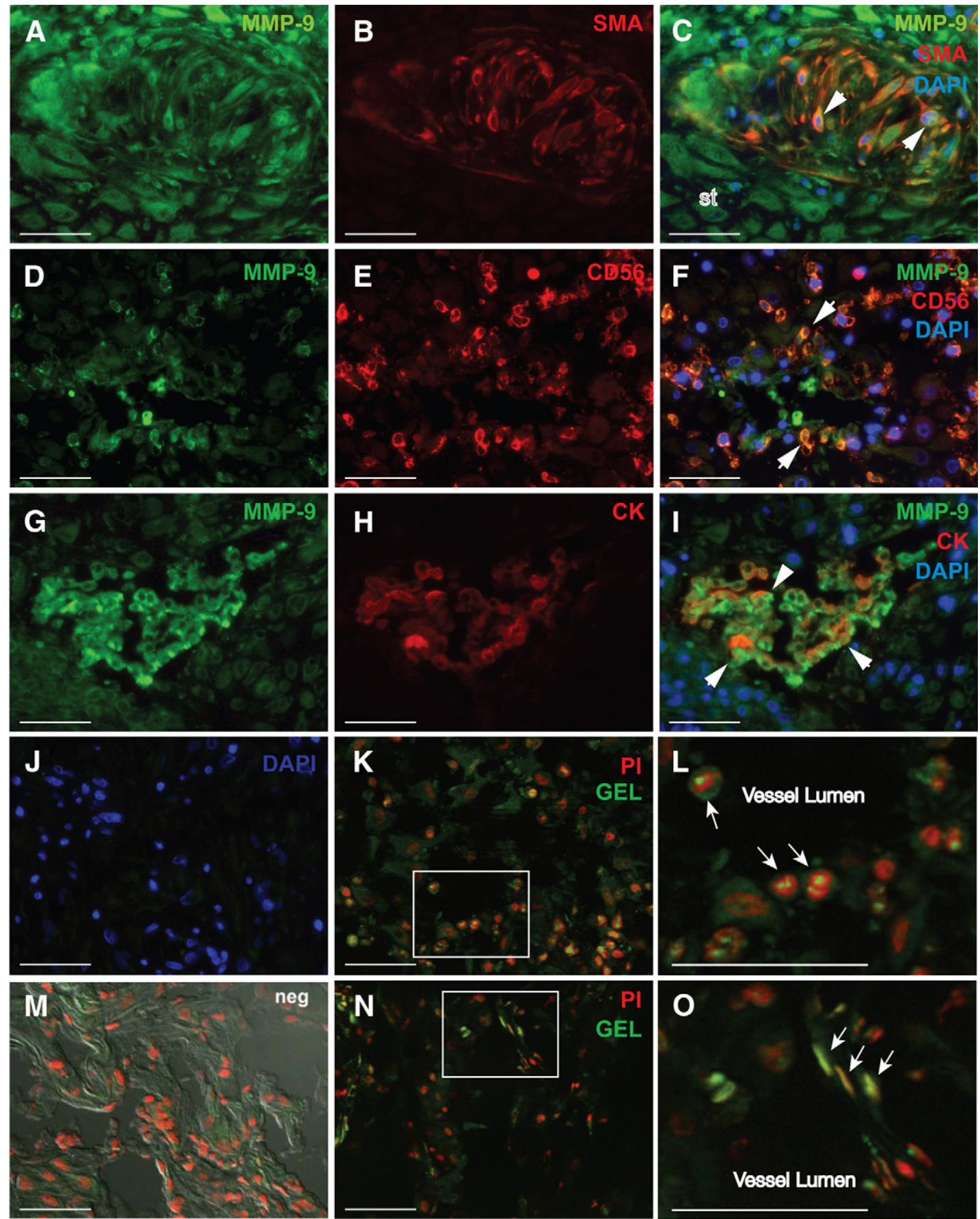

Figure 6. Matrix-metalloprotease (MMP) expression and activity in remodeling arterioles. MMP-9 (A, D, G; green) and cell markers [red: $\alpha$-SMA marking VSMC of early remodeling arterioles (B), CD56 marking uNK cells in the vascular wall of actively remodeling arterioles (E), and CK marking enEVTs of advanced remodeling arterioles $(\mathbf{H})$ ] all demonstrate colocalization (arrowheads in $\mathbf{C}, \mathbf{F}$, and $\mathbf{I}$, respectively; yellow). MMP-9 was also expressed by decidual stromal cells (st). J: Negative control for dual immunofluorescence (anti-mouse FITC and DAPI). K and N: In situ zymography revealed gelatinase reactivity (GEL: green; nuclei red) in both leukocytes (K) and VSMCs (N) that were identified in serial sections (denoted by arrows) of actively remodeling arterioles, enlarged in $\mathbf{L}$ and $\mathbf{O}$ (CD 45 and $\alpha$-SMA serial stains not shown). M: Negative control for in situ zymography. Scale bars $=50 \mu \mathrm{m}$.

lar remodeling and contribute to early decidual changes independent of inEVTs. Additionally, while decidua parietalis is used in this model to control for the role of trophoblast, which should not be present before co-culture, the decidua basalis specimens used in our previous study of in vivo remodeling also did not contain inEVTs around early remodeling arteries. It is possible that inEVTs play an important role in the later stages of vascular remodeling in the myometrial portions of spiral arteries as supported by the observation of EVTs embedded in fibrinoid in fully "physiologically transformed" vessels. ${ }^{28,29}$

Other detailed studies of early implantation sites have provided convincing evidence of subtle remodeling and VSMC disruption within decidual arteries before direct cellular contact of vascular cells with invading EVTs. ${ }^{25,30}$ These authors also described a leukocytic infiltration of 

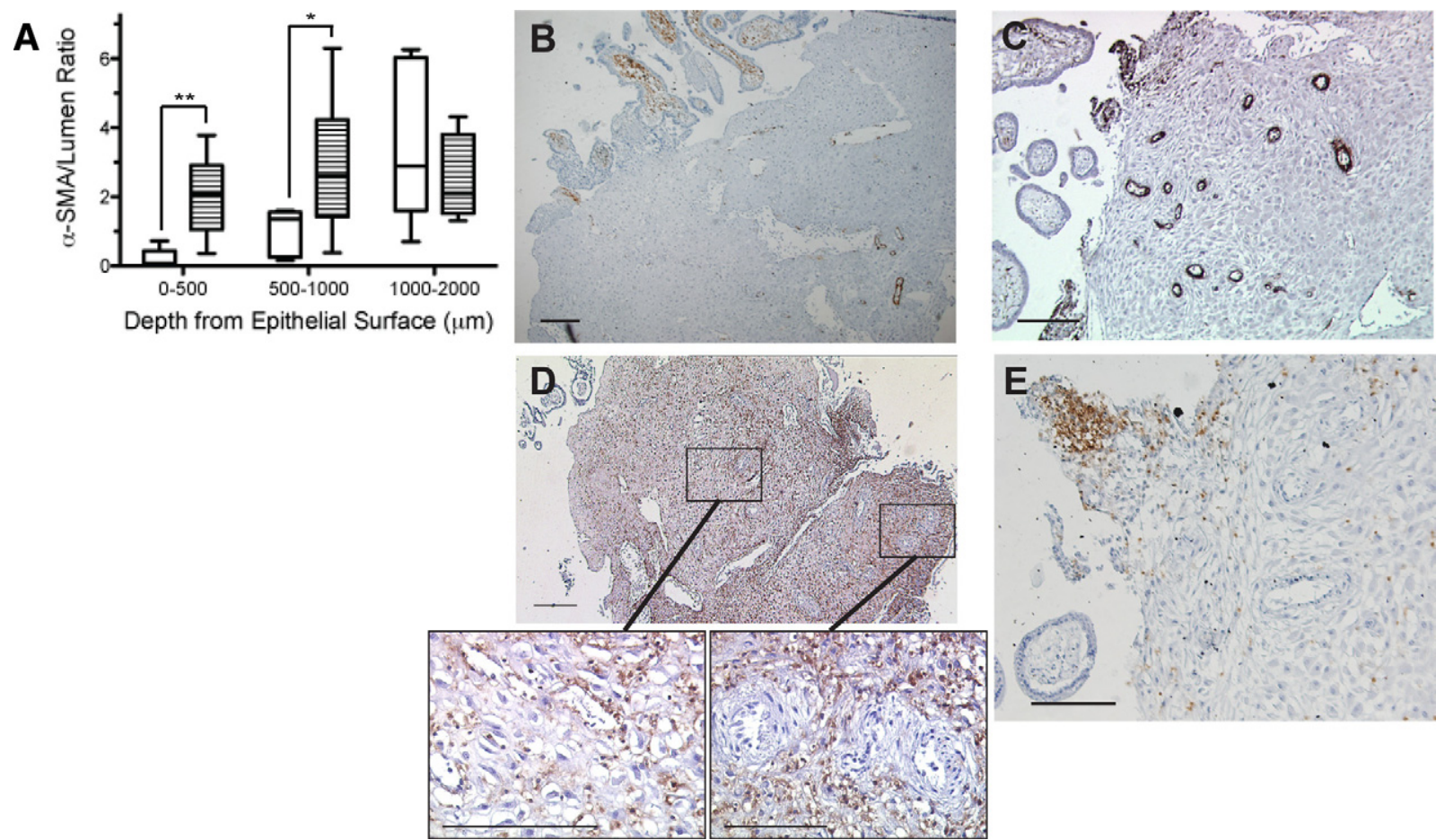

Figure 7. Inhibition of MMP-2/9 prevents decidual spiral artery remodeling. A: Quantification of $\alpha$-SMA/lumen ratios demonstrated a failure of vascular transformation in MMP-2/9 inhibited PDC (striped bars: $n=3$ ) in comparison with vehicle-injected PDC controls (white bars: $n=3$ ). Representative $\alpha$-SMA-stained images of PDCs injected with vehicle (B) or $50 \mathrm{nmol} / \mathrm{L}$ MMP-2/9 Inhibitor II (C). D: CD 45-stained vehicle-injected PDCs showing the difference in leukocyte clustering between arterioles close to placental contact and deep in the decidua. E: CD45-stained serial section of MMP-2/9 Inhibitor II-injected PDCs showing that leukocytes are not recruited to arterioles and do not invade the arterial wall. Scale bars: $200 \mu \mathrm{m}$ (B); $100 \mu \mathrm{m}(\mathbf{C}-\mathbf{E}) ; 50 \mu \mathrm{m}$ (enlarged sections from D). ${ }^{*} P<0.05,{ }^{* *} P<0.01$

decidual vessels, which was recently described as a 'decidualization-associated' vascular change. ${ }^{24}$ Similarly, in our previous work on in vivo decidua basalis arteries, we observed significant accumulation of uNK cells and macrophages in the early to mid stages of remodeling in the absence of enEVTs, but not in vessels with enEVTs present. ${ }^{15}$ The current study extends this work by clearly demonstrating that although vascular changes are observed before visible EVTs within the artery lumen, placentally-derived mechanisms are required to initiate vascular remodeling as decidua-only cultures showed no evidence of remodeling or uNK cell
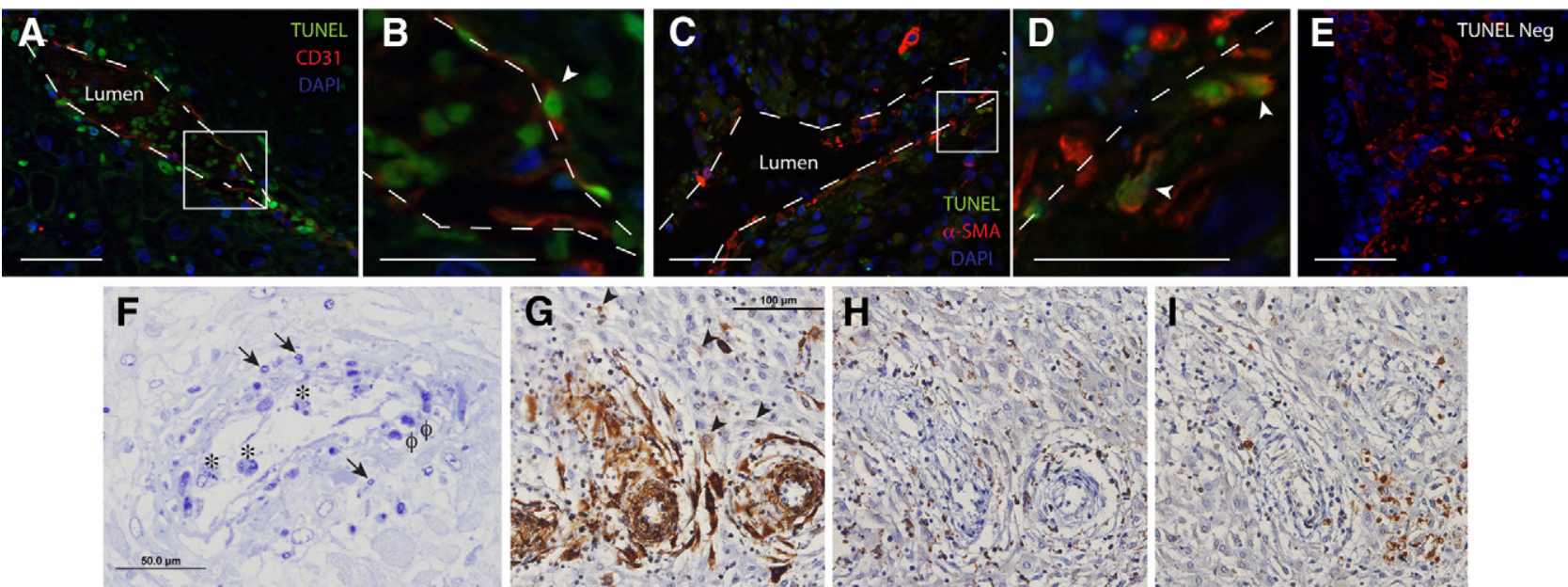

Figure 8. Mechanisms of leukocyte-mediated vascular remodeling. A: Identification of apoptotic vascular cells in actively remodeling arterioles, coincident with leukocyte infiltration, by dual immunostaining with TUNEL (green) and (A) CD31 (red) or (C) $\alpha$-SMA (red). Vessel lumens are enclosed by dotted lines. Nucle are stained with DAPI (blue). Higher resolution of dual positive apoptotic endothelial cells from A (B) and VSMCs from C (D) are shown (white arrowheads). E: Negative control for TUNEL antibody, positive for $\alpha$-SMA (red) and nuclei (blue). F: Semithin toluidine blue-stained sections of actively remodeling vessels demonstrated pyknotic apoptotic nuclei within the vascular wall (arrows), granular leukocytes (uNK cells) within the lumen (asterisk) and macrophages in the vessel wall $(\phi)(n=3)$. G-I represent a PDCs in serial section. G: Dedifferentiation of $\alpha$-SMA ${ }^{+}$VSMCs observed by loss of staining as cells "loosen" from tightly organized rings (black arrowheads). H: $\mathrm{CD}^{+} 8^{+}$macrophages. I: Lysozyme muramidase immunostaining identifies phagocytic activity in cells associated with this vessel $(n=5)$. Scale bars: $50 \mu \mathrm{m}(\mathbf{A}, \mathbf{C}, \mathbf{E}$, and $\mathbf{F}) ; 25 \mu \mathrm{m}(\mathbf{B}$ and $\mathbf{D}) ; 100 \mu \mathrm{m}(\mathbf{G}-\mathbf{I})$. 
and macrophage clustering. An example of potential placental-leukocyte signaling is the release of CXCL8 from primary EVTs stimulated with gonadotropin releasing hormone, which promotes uNK cell trans-well migration. ${ }^{31}$ The PDC model also verifies that it is the resident decidual leukocyte population that participates in remodeling as blood flow is absent and de novo leukocyte recruitment cannot occur in our model. Moreover, we suggest that enEVTs can communicate with stromal leukocytes without direct EVT-leukocyte cellular contact, potentially via changes in VSMC chemokine profiles. This hypothesis is supported by a number of studies demonstrating that VSMC and endothelium of spiral arteries express chemokines specific for macrophages and UNK cells including CCL14, 16, 21, 22, CXCL12, and CX3CR1 1. ${ }^{32,33,34}$ These recent findings, when taken together with the data presented in this manuscript, provide evidence for complex chemokine communication between EVTs, VSMCs, and decidual leukocytes at the cellular level during vascular remodeling.

Interestingly, many leukocytes are observed within the lumen of the PDC arterioles, in addition to those that penetrate the vascular wall. It is thought that the single uNK cells observed in vessel lumens and those within the vascular wall, particularly those in contact with endothelial cells, are in the process of diapedesis and infiltrate the decidual stroma from the peripheral blood. ${ }^{35}$ While this method of UNK cell recruitment may occur in vivo, the PDC model provides strong evidence that the resident decidual leukocytes can intravasate into the vasculature as remodeling occurs. This is supported by the detection of granulated metrial gland cells in the lungs of mice during pregnancy. ${ }^{36}$ Intravascular CD56 expression was localized to UNK cells and not enEVTs in the remodeling PDC arterioles, as determined by serial cytokeratin staining.

Several mechanisms have been suggested to mediate VSMC and endothelial cell loss during vascular remodeling including migration, dedifferentiation, and apoptosis. In PDCs the vascular layers of early remodeling arterioles exhibit loosening in their outermost portions and 'loosened' VSMC immunostain weaker for $\alpha$-SMA with increasing distance from the vascular wall, suggesting a loss of phenotype with cell detachment from the vascular matrix. Similar observations have been made in placental bed biopsies from early pregnancy where isolated VSMCs were found at a distance from arteries, particularly in those colonized by enEVTs. ${ }^{37}$ We suggest that this decrease in $\alpha$-SMA staining may reflect a differentiation of the VSMCs to a more myofibroblast phenotype. Similarly, the decidual stromal cells have been shown to possess plasticity and also express myofibroblast markers $\alpha$-SMA and vimentin. ${ }^{38}$ Furthermore, we have shown that active MMP-9 is highly expressed by infiltrating UNK cells, macrophages, and in actively remodeling arterioles reinforcing our previous observations, and those of others, that UNK cells associated with decidual arteries produce MMP-9. ${ }^{15,39,40}$ Secretion of MMP-9 by leukocytes within the disorganized VSMC layers of remodeling arteries may initiate vascular matrix and basement membrane destruction, allowing leuko- cytes to infiltrate, and facilitating VSMC dispersal and dedifferentiation, as is reported during vascular remodeling. ${ }^{37,41}$ Furthermore, when decidual explants from PDC were injected with an inhibitor of MMP-2/9 activity before co-culture with a placental explant, the decidual arterioles retained intact VSMC layers and displayed little or no evidence of remodeling. Loss of cellular anchoring to the extracellular matrix also triggers a form of apoptotic cell death known as anoikis ${ }^{42}$; this may represent an alternative mechanism for VSMC and endothelial apoptosis during active decidual artery remodeling. MMPs also fulfill important roles during inflammation unrelated to matrix destruction, including modulating chemokine activity via proteolytic cleavage. Of particular relevance, MMP-9 can cleave and inactivate chemokine CXCL5, while increasing CXCL8 potency in neutrophil chemoattraction. $^{31,43}$ It is possible that UNK cell-secreted MMPs increase local levels of active chemokines, such as CXCL8, providing a positive feedback mechanism by which UNK cells may stimulate their own recruitment to remodeling vessels. Interestingly, in the presence of MMP-2/9 inhibitor II leukocytes did not accumulate or infiltrate the vasculature suggesting that they were not able to respond to the initial placental stimulus and lending further support to the functional role for active MMPs in leukocyte recruitment, trophoblast invasion, and vascular remodeling. Localized MMP-9 secretion may also contribute to the tight regulation of leukocyte infiltration and cessation of local inflammation in the advanced stages of decidual remodeling.

Studies using isolated myometrial spiral arteries have demonstrated that trophoblast cells secrete cytokines from the tumor necrosis factor family, including Fasligand and tumor necrosis factor-related apoptosis-inducing ligand, which can induce apoptosis of aortic VSMC over a 24 -hour period. ${ }^{44,45}$ In contrast, some studies of placental bed biopsies have failed to identify apoptosis in perivascular cells. ${ }^{46}$ Importantly, in this article we demonstrate colocalization of TUNEL reactivity with both VSMCs and endothelial cells, coincident with early medial smooth muscle disruption and leukocyte accumulation in the PDCs. This corroborates our recent in vivo evidence of apoptotic VSMCs and endothelial cells in remodeling decidual arteries before EVT presence. ${ }^{15}$ However, the number of TUNEL ${ }^{+}$cells is unlikely to account for the full magnitude of VSMC loss. Alternatively, plasticity or dedifferentiation of VSMCs from a functional (contractile) to a synthetic (proliferative) phenotype ${ }^{47}$ may account for the extensive vascular disorganization observed in our model. VSMC susceptibility to apoptotic stimuli, and thus rapidity of vascular transformation, may also be influenced by differences between the contractile and proliferative phenotypes of VSMCs. ${ }^{48}$ Another mechanism that may contribute to the paucity of TUNEL positivity is the rapid phagocytic clearance of apoptotic cells by macrophages to minimize inflammation. Our finding of lysozyme muramidase ${ }^{+}$cells surrounding vessels with early signs of VSMC disruption supports an active role for macrophages in decidual vascular remodeling. Interestingly, UNK cells have also been shown to secrete interferon- $\gamma,{ }^{49}$ which can activate macrophages to be phagocytic $^{50}$ and may also function to recruit macrophages to 
the remodeling arteries. These mechanisms suggest participation of UNK cells and macrophages in VSMC disruption, death, and clearance in remodeling.

These results extend our understanding of the temporal sequence of events and mechanisms of remodeling, strongly supporting an integral role for uNK cells and macrophages in decidual vascular transformation. As demonstrated by the failure of vascular remodeling induced by inhibition of MMP-2/9 activity, we further support the body of literature that implicates these proteases in maintenance and progression of healthy pregnancy. Furthermore, these experiments demonstrate how the PDC model can be manipulated to investigate the role of regulatory factors, such as cytokines, proteases, or hormones, in mediating vascular remodeling. Study of the normal physiological process of human decidual spiral artery transformation is critical to identify causative factors of impaired vascular remodeling and reduced utero-placental perfusion associated with preeclampsia and intrauterine growth restriction.

\section{Acknowledgments}

We thank the donors, the Research Centre for Women's and Infants' Health BioBank Program of the Canadian Institutes of Health Research Group in Development and Fetal Health, the Samuel Lunenfeld Research Institute, and the Mount Sinai Hospital/University Health Network Department of Obstetrics \& Gynecology for the human specimens used in this study (Mount Sinai Hospital Research Ethics Board 04-0018-U). We also thank Colleen Butchart and the nursing staff at St. Mary's Hospital in Manchester for their invaluable help with subject recruitment and tissue collection (ethical approval from North West REC \#08/H1010/28).

\section{References}

1. Dunk C, Huppertz B, Kingdom J: Development of the placenta and its circulation. Edited by Rodeck $\mathrm{CH}$, Whittle MJ. London, Churchill Livingstone Elsevier. 2009, pp. 69-96

2. Brosens JJ, Pijnenborg R, Brosens IA: The myometrial junctional zone spiral arteries in normal and abnormal pregnancies: a review of the literature. Am J Obstet Gynecol 2002, 187:1416-1423

3. Benirschke K, Kaufmann P, Baergen R: Pathology of the Human Placenta. New York, NY, Springer, 2006

4. Bulmer JN. Immune cells in decidua. Edited by Kurpisz M, Fernandez N. Oxford, Oxford Bios Scientific Publishers. 1995, 313-334

5. King A, Burrows T, Verma S, Hiby S, Loke YW: Human uterine lymphocytes. Hum Reprod Update 1998, 4:480-485

6. Hanna J, Goldman-Wohl D, Hamani Y, Avraham I, Greenfield C, Natanson-Yaron S, Prus D, Cohen-Daniel L, Arnon TI, Manaster I, Gazit R, Yutkin V, Benharroch D, Porgador A, Keshet E, Yagel S, Mandelboim O: Decidual NK cells regulate key developmental processes at the human fetal-maternal interface. Nat Med 2006, 12:1065-1074

7. Tabiasco J, Rabot M, Aguerre-Girr M, El Costa H, Berrebi A, Parant O, Laskarin G, Juretic K, Bensussan A, Rukavina D, Le Bouteiller P: Human decidual NK cells: unique phenotype and functional properties - a review. Placenta. 2006, 27 (Suppl A):S34-S39

8. Davies MJ: Pathology of arterial thrombosis. Br Med Bull 1994, 50:789-802

9. Porta C, Subhra Kumar B, Larghi P, Rubino L, Mancino A, Sica A: Tumor promotion by tumor-associated macrophages. Adv Exp Med Biol 2007, 604:67-86
10. Croy BA, Di Santo JP, Greenwood JD, Chantakru S, Ashkar AA: Transplantation into genetically alymphoid mice as an approach to dissect the roles of uterine natural killer cells during pregnancy-a review. Placenta. 2000, 21 (Suppl A):S77-S80

11. Croy BA, Esadeg $S$, Chantakru S, van den Heuvel M, Paffaro VA, He $\mathrm{H}$, Black GP, Ashkar AA, Kiso Y, Zhang J: Update on pathways regulating the activation of uterine Natural Killer cells, their interactions with decidual spiral arteries and homing of their precursors to the uterus. J Reprod Immunol 2003, 59:175-191

12. Ashkar AA, Black GP, Wei Q, He H, Liang L, Head JR, Croy BA: Assessment of requirements for IL-15 and IFN regulatory factors in uterine NK cell differentiation and function during pregnancy. J Immunol 2003, 171:2937-2944

13. Zhang $\mathrm{JH}, \mathrm{He} \mathrm{H}$, Borzychowski AM, Takeda K, Akira S, Croy BA: Analysis of cytokine regulators inducing interferon production by mouse uterine natural killer cells. Biol Reprod 2003, 69:404-411

14. King A, Hiby SE, Gardner L, Joseph S, Bowen JM, Verma S, Burrows TD, Loke YW: Recognition of trophoblast HLA class I molecules by decidual NK cell receptors-a review. Placenta. 2000, 21 (Suppl A):S81-S85

15. Smith SD, Dunk CE, Aplin JD, Harris LK, Jones RL: Evidence for immune cell involvement in decidual spiral arteriole remodeling in early human pregnancy. Am J Pathol 2009, 174:1959-1971

16. Lash GE, Otun HA, Innes BA, Bulmer JN, Searle RF, Robson SC Inhibition of trophoblast cell invasion by TGFB1, 2, and 3 is associated with a decrease in active proteases. Biol Reprod 2005, 73:374-381

17. Bischof $\mathrm{P}$, Meisser A, Campana A: Control of MMP-9 expression at the maternal-fetal interface. J Reprod Immunol 2002, 55:3-10

18. Cohen M, Meisser A, Bischof P: Metalloproteinases and human placental invasiveness. Placenta 2006, 27:783-793

19. Salamonsen LA: Matrix metalloproteinases and endometrial remodelling. Cell Biol Int 1994, 18:1139-1144

20. Handsley MM, Edwards DR: Metalloproteinases and their inhibitors in tumor angiogenesis. Int J Cancer 2005, 115:849-860

21. Coussens LM, Tinkle CL, Hanahan D, Werb Z: MMP-9 supplied by bone marrow-derived cells contributes to skin carcinogenesis. Cell 2000, 103:481-490

22. Vicovac L, Jones CJ, Aplin JD: Trophoblast differentiation during formation of anchoring villi in a model of the early human placenta in vitro. Placenta 1995, 16:41-56

23. Dunk C, Petkovic L, Baczyk D, Rossant J, Winterhager E, Lye S: A novel in vitro model of trophoblast-mediated decidual blood vessel remodeling. Lab Invest 2003, 83:1821-1828

24. Pijnenborg R, Vercruysse L, Hanssens M: The uterine spiral arteries in human pregnancy: facts and controversies. Placenta 2006 , 27:939-958

25. Pijnenborg R, Bland JM, Robertson WB, Brosens I: Uteroplacental arterial changes related to interstitial trophoblast migration in early human pregnancy. Placenta 1983, 4:397-413

26. Moser G, Theuerkauf RS, Flieser N, Huppertz B. Mononuclear extravillous trophoblast cells do not penetrate the decidual epithelium in a double tissue confrontation assay. Placenta 2008, 29:A.88

27. Harris JWS, Ramsey EM: The morphology of human uteroplacental vasculature. Carnegie Inst. Contrib Embryol 1966, 38:43-58

28. Brosens I, Robertson WB, Dixon HG: The physiological response of the vessels of the placental bed to normal pregnancy. J Pathol Bacteriol 1967, 93:569-579

29. Pijnenborg R, Bland JM, Robertson WB, Dixon G, Brosens I: The pattern of interstitial trophoblastic invasion of the myometrium in early human pregnancy. Placenta 1981, 2:303-316

30. Craven CM, Morgan T, Ward K: Decidual spiral artery remodelling begins before cellular interaction with cytotrophoblasts. Placenta 1998, 19:241-252

31. Cavanagh PC, Dunk C, Pampillo M, Szereszewski JM, Taylor JE, Kahiri C, Han V, Lye S, Bhattacharya M, Babwah AV: Gonadotropinreleasing hormone-regulated chemokine expression in human placentation. Am J Physiol Cell Physiol 2009, 297:C17-C27

32. Jones RL, Hannan NJ, Kaitu'u TJ, Zhang J, Salamonsen LA: Identification of chemokines important for leukocyte recruitment to the human endometrium at the times of embryo implantation and menstruation. J Clin Endocrinol Metab 2004, 89:6155-6167

33. Hannan NJ, Jones RL, White CA, Salamonsen LA: The chemokines. 
CX3CL1, CCL14, and CCL4, promote human trophoblast migration at the feto-maternal interface Biol Reprod 2006, 74:896-904

34. Smith SD, Aplin JD, Harris LK, Dunk C, Jones RL: Potential involvement of chemokines in recruiting UNK cells and macrophages to remodeling spiral arteries. Reproductive Sciences. 2009, 16:110A

35. Kruse A, Martens N, Fernekorn U, Hallmann R, Butcher EC: Alterations in the expression of homing-associated molecules at the maternal/fetal interface during the course of pregnancy. Biol Reprod 2002, 66:333-345

36. Stewart I: Granulated metrial gland cells in the lungs of mice in pregnancy and pseudopregnancy. J Anat 1985, 140(Pt 4):551-563

37. Lash GE, Innes BA, Robson SC, Bulmer JN. Trophoblast increases the distance vascular smooth muscle cells move from the wall of transforming spiral arteries. Placenta 2008, 29:A.16

38. Kimatrai M, Oliver C, Abadia-Molina AC, Garcia-Pacheco JM, Olivares EG: Contractile activity of human decidual stromal cells. J Clin Endocrinol Metab 2003, 88:844-849

39. Naruse K, Lash GE, Innes BA, Otun HA, Searle RF, Robson SC, Bulmer JN: Localization of matrix metalloproteinase(MMP)-2. MMP-9 and tissue inhibitors for MMPs (TIMPs) in uterine NK cells in early human pregnancy. Hum Reprod 2009, 24:553-561

40. Jones RL, Findlay JK, Farnworth PG, Robertson DM, Wallace E, Salamonsen LA: Activin $A$ and inhibin $A$ differentially regulate human uterine matrix metalloproteinases: potential interactions during decidualization and trophoblast invasion. Endocrinology 2006, 147:724-732

41. Whitley GS, Cartwright JE: Trophoblast-mediated spiral artery remodelling: a role for apoptosis. J Anat 2009, 215:21-26

42. Ingber DE: Mechanical signaling and the cellular response to extracellular matrix in angiogenesis and cardiovascular physiology. Circ Res 2002, 91:877-887
43. Van Den Steen PE, Wuyts A, Husson SJ, Proost P, Van Damme J, Opdenakker G: Gelatinase B/MMP-9 and neutrophil collagenase/ MMP-8 process the chemokines human GCP-2/CXCL6. ENA-78/ CXCL5 and mouse GCP-2/LIX and modulate their physiological activities Eur J Biochem 2003, 270:3739-3749

44. Keogh RJ, Harris LK, Freeman A, Baker PN, Aplin JD, Whitley GS, Cartwright JE: Fetal-derived trophoblast use the apoptotic cytokine tumor necrosis factor-alpha-related apoptosis-inducing ligand to induce smooth muscle cell death. Circ Res 2007, 100:834-841

45. Harris LK, Keogh RJ, Wareing M, Baker PN, Cartwright JE, Aplin JD, Whitley GS: Invasive trophoblasts stimulate vascular smooth muscle cell apoptosis by a fas ligand-dependent mechanism. Am J Pathol 2006, 169:1863-1874

46. Bulmer JN, Innes BA, Lash GE, Robson SC: Transformation of uterine spiral arteries in normal human pregnancy: no in-situ evidence for apoptosis of vascular smooth muscle cells. Toronto, Canada, Elsevier, 2006, p181A

47. Stegemann JP, Hong H, Nerem RM: Mechanical, biochemical, and extracellular matrix effects on vascular smooth muscle cell phenotype. J Appl Physiol 2005, 98:2321-2327

48. Su BY, Shontz KM, Flavahan NA, Nowicki PT: The effect of phenotype on mechanical stretch-induced vascular smooth muscle cell apoptosis. J Vasc Res 2006, 43:229-237

49. Ashkar AA, Di Santo JP, Croy BA: Interferon gamma contributes to initiation of uterine vascular modification, decidual integrity, and uterine natural killer cell maturation during normal murine pregnancy. J Exp Med 2000, 192:259-270

50. McIntire RH, Ganacias KG, Hunt JS: Programming of human monocytes by the uteroplacental environment. Reprod Sci 2008, 15:437-447 